\title{
Selective enhancement of attentional networks in college table tennis athletes: a preliminary investigation
}

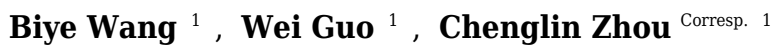 \\ ${ }^{1}$ Department of Sport Psychology, School of Kinesiology, Shanghai University of Sport, Shanghai, China \\ Corresponding Author: Chenglin Zhou \\ Email address: chenglin_zhou@126.com
}

The purpose of the study was to investigate the characteristics of the attentional network in college table tennis athletes. A total of 65 college students categorized as table tennis athlete group or non-athlete group participated in the study. All participants completed the attentional network test (ANT) which measured the alerting, orienting and executive control networks. The results showed a significant difference between the athlete and nonathlete group for executive control network $(p<0.01$ ), while no differences were observed for alerting $(p>0.05)$ or orienting $(p>0.05)$ networks. These results combined suggest that college table tennis athletes exhibited selectively enhanced executive control of attentional networks. 


\title{
Selective Enhancement of Attentional Networks in College Table Tennis
}

\section{Athletes: A Preliminary Investigation}

\author{
Biye Wang, Wei Guo, Chenglin Zhou* \\ Department of Sport Psychology, School of Kinesiology, Shanghai University of Sport, \\ Shanghai, China \\ *Corresponding author: Chenglin Zhou \\ Email: chenglin_zhou@126.com
}

\section{Abstract}

The purpose of the study was to investigate the characteristics of the attentional network in college table tennis athletes. A total of 65 college students categorized as table tennis athletes or non-athletes participated in the study. All participants completed the attentional network test (ANT) which measured the alerting, orienting and executive control networks. The results showed a significant difference between the athlete and nonathlete group for executive control network $(p<0.01)$, while no differences were observed for alerting $(p>0.05)$ or orienting ( $p>0.05)$ networks. These results combined suggest that college table tennis athletes exhibited selectively enhanced executive control of attentional networks.

\section{Introduction}

The ability to selectively focus on the relevant information while ignoring irrelevant information is a basic function of our brain to ensure that we can interact with the environment effectively. This ability requires attention, which is a core function of cognitive system and regulates other cognitive functions such as memory and language (Posner \& Petersen, 1990). More specifically, attention plays an important role in sports (Williams \& Davids, 1999). Obviously, it is crucial for most athletes to choose the important information to process in an extreme short period of time in a competition context (Allard, Brawley, Deakin, \& Elliot, 1989). And it would be difficult to achieve any goals for athletes with easily disturbed attention. Thus, it is reasonable to speculate that the sport-specific attentional function may develop better in athletes, relative to non-athletes. However, it is still unclear whether the athletes may have a better general attentional function (Voss, Kramer, Basak, Prakash, \& Roberts, 2010). So the present study focused on the transfer of sport-specific attentional function to general attentional function. Indeed, several studies have already focused on the possible relationship between athlete experience and general attentional function in a laboratory setting (Enns \& Richards, 1997; Memmert, 2009; 
Memmert, Simons, \& Grimme, 2009; Nougier, Azemar, Stein, \& Ripoll, 1992). However, these kind of studies yielded mixed results due to variation in laboratory attentional tasks (Voss et al., 2010). The attentional network test (ANT) developed by Fan et al. (2002) is one of the most dominant attention paradigms and seems to be appropriate for this kind of study (Fan, McCandliss, Sommer, Raz, \& Posner, 2002). It is a short and simple computerized task that measures the attentional networks independently. The task was based on the well-known attention network theory proposed by Posner and Petersen (Petersen \& Posner, 2012; Posner \& Petersen, 1990). According to this theory, the attention system could be divided into three different networks: alerting network, orienting network and executive control network. Each of them representing a set of certain attentional functions and little overlap between the three networks was revealed by a neuroimaging analysis (Fan, McCandliss, Fossella, Flombaum, \& Posner, 2005). The alerting network is related to maintenance of certain levels of arousal and sustained vigilance, the orienting network allows selection of information from multiple sensory inputs, and the executive control network is related to the ability to monitor and resolve conflict (Petersen \& Posner, 2012; Posner \& Petersen, 1990).

Although few studies have explored the three attentional networks of athletes in one experiment using the ANT, there is some evidence showing the characteristics of alerting, orientation or executive control in athletes in different studies. The alerting and orientation ability of athletes is mainly measured by the spatial cueing paradigm (Posner \& Fan, 2008). For example, Enns and Richards (1997) used different cue-target intervals to investigate the alerting effect. The results revealed that athletes sustained a high level of alertness over the longest cue-target interval (Enns \& Richards, 1997). Cereatti et al. (2009) observed athletes outperform non-athletes on the voluntary orientation of attention (Cereatti, Casella, Manganelli, \& Pesce, 2009). Studies have also demonstrated athletes to exhibit higher proficiency on tasks testing executive function (Jacobson \& Matthaeus; 2014; Vestberg, Gustafson, Maurex, Ingvar, \& Petrovic, 2012; Verburgh, Scherder, van Lange, \& Oosterlaan, 2014). For example, Jocobson and Matthaeus (2014) revealed that athletes performed better than non-athletes on a problem solving as well as an inhibition task, suggesting that athletes achieved better executive control ability.

This study was designed to investigate the association between sports training experiences and the modulation of attentional network functions. It could, to some extent, answer a basic question in brain plasticity research whether an individual's experience can affect the attentional process. Athletes are one of the most suitable models to investigate this question because of their unique experience. Compared with non-athletes, most of them trained with larger amount regularly for several years. Although it seems that previous studies have already focused on this topic for decades, the present study and these studies differ in many aspects. Firstly, athletes from one of the typical open-skilled sports, table tennis, served as the athlete group in this study. Previous studies mainly explored the attentional function of athletes from closed-skill sports (e.g. swimming, running) rather than athletes from open-skilled sports (e.g. tennis, table tennis) (Voss et al., 2010). Compared to closedskill sports, open-skill sports require individuals to invest higher cognitive effort in the unpredictable environment which may serve as cognitive training to enhance the attention skill (Tang \& Posner, 2009). It has been shown that open-skill athletes are more flexible in visual attention, decision making, inhibition, and working memory, compared to closed-skill athletes (Voss et al., 2010; Wang et al., 2013; Heppe, Kohler, Fleddermann, \& Zentgraf, 2016). Secondly, the attentional network test (ANT) was adopted in this study to evaluate the efficiencies of the three attention networks in one experiment, it is more efficient than the battery of attention test mainly used in previous studies because the ANT requires only about 15 min to complete, and 
there are very little overlaps among the three networks. It has been widely used in certain clinical populations, however few studies have investigated the differences between athletes and non-athletes on the ANT. To the best of our knowledge, this is the first study to investigate the characteristics of table tennis athlete's attentional networks with the ANT.

The present study aimed to investigate the characteristics of the attentional network in college table tennis athletes using the ANT. Although previous studies have indicated that chronic exercise (Pérez, Padilla, Parmentier, \& Andrés, 2013) and acute exercise (Chang, Pesce, Chiang, Kuo, \& Fong, 2015) improve the performance on ANT in non-athletes, this was the first study to our knowledge to adopt table tennis athletes as the participants. There are three reasons for choosing table tennis athletes as the participants. Firstly, table tennis is one of the fastest ball sports and the response window dictated by the ball speed is very brief. The table tennis athletes have to use advanced cues to decide what response is required as soon as possible (Padulo et al., 2015), and therefore, they would develop superior alerting and orienting ability. Secondly, table tennis is a highly developed tactical skill, involving creativity, concentration, competitiveness, apprehension, self-regulation, and will power (Raab, Masters, \& Maxwell, 2005). Table tennis athletes compete in a dynamically changing, unpredictable, and externally-paced environment which may lead to better executive control ability. Thirdly, table tennis is one of the most popular sports in China. Table tennis athletes are trained systematically and have a high competition level, so they are the perfect samples to investigate the relationship between athlete training experience and attentional function. Based on the results of previews studies which focused on the three networks of attention separately, it was hypothesized that athletes would perform better on the alerting, orientation and executive network than non-athletes.

\section{Method}

\subsection{Participants}

A total of 65 individuals categorized as athletes or non-athletes participated in the study. They were recruited through advertisements posted in the campus of Shanghai University of Sport. The athlete group was composed of 31 table tennis players (mean age $=21.9$, ranging from 19 to 25,11 females) whom satisfied all of the following criteria: (1) had 5 or more years of professional training experience, (2) qualified as the National Player at Second Grade or above, (3) trained more than three times a week in the last 2 years, (4) trained for 2 or more hours each time. The non-athlete group was composed of 35 students (mean age $=21.9$, ranging from 19 to 25,14 females) majoring in psychology or kinesiology. The non-athlete group matched the athlete group in age and education, but they had no experience of playing table tennis, nor any experience of athlete training. The non-athlete group had a moderate physical activity level which was measured by the Taiwan version of the International Physical Activity Questionnaire (IPAQ) (Liou, Jwo, Yao, Chiang, \& Huang, 2008). All the participants were right-handed and had normal or corrected to normal visual acuity. No individuals reported having a history of neurological or psychiatric disorder. Written informed consent was obtained from each participant prior to the study. All participants received a payment of approximately $\$ 10$ for taking part in the experiment. Table 1 shows the main characteristics of the subjects. This study was approved by the Ethics 
110

112

113

114

115

116

117

118

119

120

121

122

123

124

125

126

127

128

129

130

131

132

133

134

135

136

137

138

\subsection{Attention network test}

Attention network test (ANT) was designed to assess the function of the three different attention networks (Fan et al., 2002). A fixation cross was presented in the center of a computer screen at the onset of each trial. After a random interval of 400 to $1600 \mathrm{~ms}$, cues would present in one of the four possible conditions: no cue, center cue (the fixation cross was replaced by an asterisk), double cue (two asterisks were respectively displayed above and below the fixation cross), or spatial cue (an asterisk were displayed either above or below the fixation cross). The cues remained visible for $100 \mathrm{~ms}$. The presentation of asterisks provided temporal information about the appearance of target stimuli. The asterisk in the spatial cue condition provided additional information about the location of target stimuli. The spatial cues were always valid. The fixation cross was displayed alone for $400 \mathrm{~ms}$ after the disappearance of cue. Then a target stimulus was presented above or below the fixation cross according to the indication of the previous cue. The target stimulus consisted of five horizontally arranged arrows or lines. Participants were required to press the corresponding key to indicate the direction of the central target arrow. The other four arrows or lines served as flankers in the task with three possible conditions: congruent condition (arrows pointed in the same direction as the central arrow), incongruent condition (arrows pointed in the opposite direction of the central arrow), or neutral condition (lines with no direction information). The target stimulus remained on the screen until the participant responded or for $1700 \mathrm{~ms}$ if no answer was given.

The participants were instructed to concentrate on the fixation cross throughout the task. A numeric keyboard was placed in front of the participant and the participant was required to lightly put his left hand index finger on key " 1 " and right hand index finger on key "3". Once target stimuli were presented, participants were instructed to respond as fast and accurately as possible by pressing the key "1" for left directed central target arrow and pressing the key " 3 " when the direction was right.

Four blocks were included in this test. Each block contained 48 trials based on the combination of four cues conditions (no cue, center cue, double cue, and spatial cue), three flankers' conditions (congruent, incongruent, and neutral), two directions (left or right directed target arrow) and two locations (target displayed above or below the fixation cross). Each trial was presented only once in a block. The stimuli were presented and the data were recorded using Psychtoolbox (Brainard, 1997) (see Fig.1).

The three components of attentional network were computed as follows: no cue RTs versus double cue RTs for alerting, central cue RTs versus spatial cue RTs for orienting and congruent flankers RTs versus incongruent flankers RTs for executive network. 


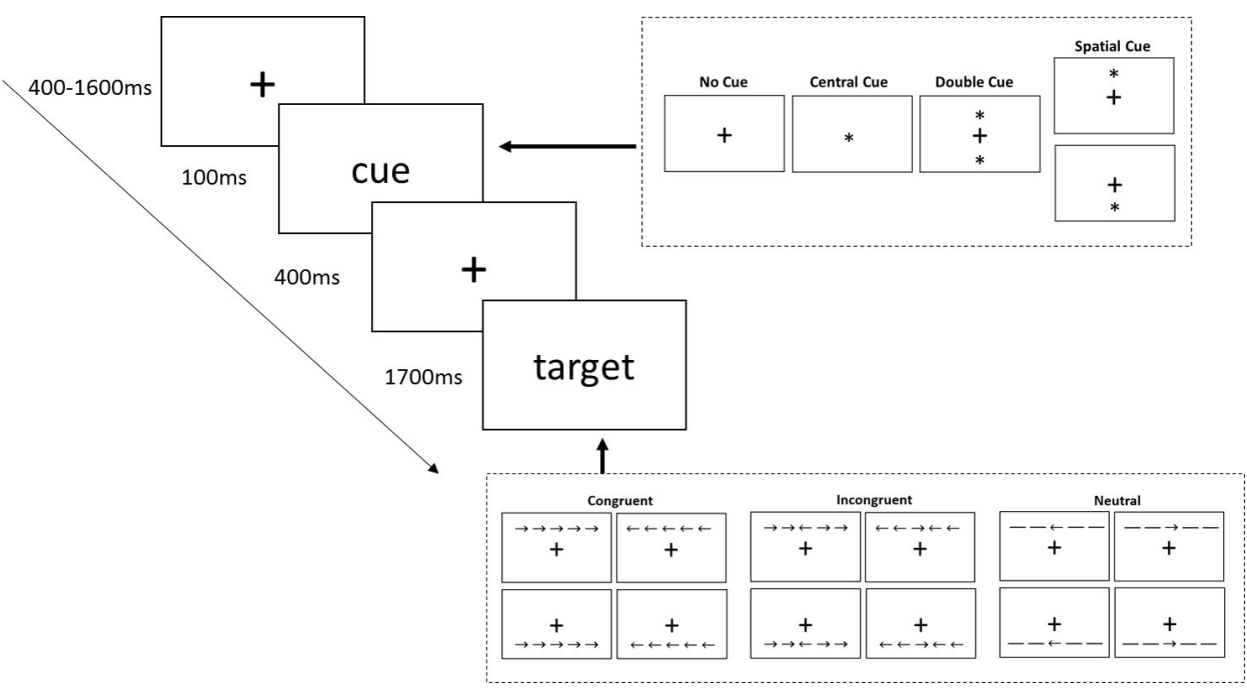

Fig. 1 Stimuli and experimental paradigm of Attention Network Test (ANT)

\subsection{Procedure}

As a requirement of the advertisements, all the participants had to contact the researchers by telephone first. A survey about the demographic data of participants was conducted during the call. Athlete participants were further asked about their training experiences. Participants who met the criteria (see 2.1 Participants) were invited to our laboratory on another day to participate in the experiment. They were instructed to abstain from alcohol for 24 hours and from caffeine-containing substances for 12 hours before the experiment.

After arriving at the laboratory, participants were asked to sign an informed consent form and were assessed by the Taiwan version of the International Physical Activity Questionnaire (IPAQ). Then the purpose of the study and the instruction of ANT were introduced to them in written form. After participants reported understanding the instructions, they performed the ANT task individually in a dimly lit and quiet room. At first, they had to perform a practice block with 24 random trials. If their response accuracy reached $80 \%$, they could perform their next 4 experimental blocks of 48 trials in each; otherwise, they would perform another practice block until their accuracy reached $80 \%$. Participants were allowed to rest between each block, and they could start the next block by pressing any keys once they felt adequately rested. Completing the whole task required about 17 minutes, including both practice and experimental blocks.

\subsection{Design and statistical analysis}

A mixed factors design was adopted in the study. The athlete and non-athlete group was a between-subjects variable, the cue type (no cue, central cue, double cue and spatial cue) and flankers type (neutral, congruent, incongruent) were within-subject variables. The dependent variables were response times (RTs) and accuracy rates. They were analyzed with a 2 (group) $\times 4$ (cue type $) \times 3$ (flanker type ) mixed-design ANOVA.

A t-test between athlete and non-athlete groups was carried out in order to explore the effect of athlete 
163

164

165

166

167

168

169

170

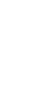

experience on each component of attentional network.

\section{Results}

\subsection{Participant characteristics}

No significant differences were observed in age $\left(\mathrm{F}_{(1,63)}=0.00, \mathrm{p}=0.98\right)$, height $\left(\mathrm{F}_{(1,63)}=3.29, \mathrm{p}=0.07\right)$, weight $\left(\mathrm{F}_{(1,63)}=2.92, \mathrm{p}=0.09\right)$, BMI $\left(\mathrm{F}_{(1,63)}=0.64, \mathrm{p}=0.43\right)$, average reaction time $\left(\mathrm{F}_{(1,63)}=1.45, \mathrm{p}=0.23\right)$ and accuracy rate $\left(\mathrm{F}_{(1,63)}=0.07, \mathrm{p}=0.79\right)$, and as expected, a significant difference was observed in physical activity level (overall score on IPAQ) $\left(\mathrm{F}_{(1,63)}=4.29, \mathrm{p}<0.05\right)$ of the two groups (see Table.1).

Table.1 The main characteristics of the subjects in different groups

\begin{tabular}{|c|c|c|}
\hline & athlete group $(n=31)$ & non-athlete group $(n=34)$ \\
\hline Female & 11 & 14 \\
\hline Age (yr) & $21.90 \pm 1.72$ & $21.91 \pm 1.80$ \\
\hline Height (cm) & $1.73 \pm 0.08$ & $1.69 \pm 0.10$ \\
\hline Weight (kg) & $65.18 \pm 9.38$ & $61.13 \pm 9.67$ \\
\hline $\operatorname{BMI}\left(\mathrm{kg} / \mathrm{m}^{2}\right)$ & $21.69 \pm 1.72$ & $21.32 \pm 1.95$ \\
\hline \multicolumn{3}{|l|}{ IPAQ (METs/week) } \\
\hline Vigorous (METs/week) & $3587.09 \pm 2372.72$ & $2037.65 \pm 5109.58$ \\
\hline Moderate (METS/week) & $1597.42 \pm 1659.15$ & $927.06 \pm 1386.74$ \\
\hline Walking (METs/week) & $1448.47 \pm 1763.65$ & $1297.68 \pm 1261.23$ \\
\hline Overall (METs/week) & $6632.99 \pm 3808.16$ & $4262.38 \pm 5229.69^{*}$ \\
\hline Reaction time (ms) & $475.88 \pm 48.43$ & $488.45 \pm 34.94$ \\
\hline Accuracy $(\%)$ & $97.93 \pm 1.93$ & $98.05 \pm 1.68$ \\
\hline
\end{tabular}

Note. BMI=body mass index, IPAQ=International Physical Activity Questionnaire, METs=metabolic equivalents.

\subsection{Mean RTs}

For the RTs analysis, an outlier correction was done by excluding the trials which were 3 standard deviations from the mean for each flanker condition (congruent, incongruent and neutral) individually. The method of outlier correction was suggested by one reviewer. The wrong trials were also excluded, and the proportion of excluded data was $1.1 \%$. Results showed a significant main effect of cue type (F $(3,189)$ $\left.=138.82, \mathrm{p}<0.01, \eta_{\mathrm{p}}{ }^{2}=0.70\right)$, the RTs were the longest in the no cue condition, and the shortest in the spatial cue condition. A significant main effect also observed in flanker type $\left(F_{(2,126)}=318.31, p<0.01, \eta_{p}{ }^{2}=0.84\right)$. The RTs were longer in the incongruent condition than in the congruent or neutral condition. Furthermore, there were significant interactions between flanker type and cue type $\left(F_{(6,378)}=7.90, p<0.01, \eta_{p}{ }^{2}=0.11\right)$, group and flanker type $\left(\mathrm{F}_{(2,126)}=4.68, \mathrm{p}<0.01, \eta_{\mathrm{p}}{ }^{2}=0.07\right)$. The interaction contrasts for flanker type and cue type revealed significant differences between the congruent and incongruent conditions, incongruent conditions and neutral 
193

194

conditions under all cue conditions, no significant differences were observed between congruent and neutral conditions under all cue conditions. The interaction contrast for the group and flanker type revealed significant differences between the groups under incongruent condition, no significant differences were observed between the groups under congruent and neutral conditions. There were no significant main effect of group $\left(\mathrm{F}_{(1,63)}\right.$ $\left.=1.45, \mathrm{p}=0.23, \eta_{\mathrm{p}}{ }^{2}=0.02\right)$, group $\times$ cue type $\left(\mathrm{F}_{(3,189)}=0.63, \mathrm{p}=0.60, \eta_{\mathrm{p}}{ }^{2}=0.01\right)$ or group $\times$ cue type $\times$ flanker type $\left(\mathrm{F}_{(6,378)}=0.41, \mathrm{p}=0.87, \eta_{\mathrm{p}}{ }^{2}=0.00\right)$ interaction. The description data of the mean RTs and standard deviations of athlete and non-athlete group according to the cue and flanker type are shown in Table.2.

Table.2 Mean RTs (ms) and standard deviations of athlete and non-athlete group according to cue and flanker type

\begin{tabular}{ccccccc}
\hline & \multicolumn{2}{c}{ Congruent } & \multicolumn{2}{c}{ Incongruent } & \multicolumn{2}{c}{ Neutral } \\
\cline { 2 - 6 } & Athlete & Non-athlete & Athlete & Non-athlete & Athlete & Non-athlete \\
\hline No cue & $477.3 \pm 50.8$ & $486.1 \pm 36.2$ & $532.6 \pm 55.2$ & $554.0 \pm 49.4$ & $485.6 \pm 55.3$ & $492.0 \pm 42.9$ \\
Central cue & $455.8 \pm 49.0$ & $459.8 \pm 38.6$ & $520.2 \pm 63.5$ & $537.3 \pm 43.1$ & $458.4 \pm 49.4$ & $465.6 \pm 40.7$ \\
Double cue & $459.7 \pm 49.7$ & $465.0 \pm 41.1$ & $519.6 \pm 56.3$ & $542.0 \pm 43.5$ & $457.7 \pm 48.7$ & $467.4 \pm 43.2$ \\
Spatial cue & $434.0 \pm 52.0$ & $446.2 \pm 36.5$ & $472.3 \pm 51.6$ & $501.4 \pm 43.8$ & $435.9 \pm 48.1$ & $443.4 \pm 36.9$ \\
\hline
\end{tabular}

\subsection{Accuracy}

For the accuracy analysis, significant main effects of cue type $\left(\mathrm{F}_{(3,189)}=7.89, \mathrm{p}<0.01, \eta_{\mathrm{p}}{ }^{2}=0.11\right)$, and flanker type $\left(\mathrm{F}_{(2,126)}=39.9, \mathrm{p}<0.01, \eta_{\mathrm{p}}{ }^{2}=0.39\right)$ were revealed. Furthermore, there were significant interactions between flanker type and cue type $\left(\mathrm{F}_{(6,378)}=4.10, \mathrm{p}<0.01, \eta_{\mathrm{p}}{ }^{2}=0.06\right)$. Interaction contrast revealed significant differences between the congruent and incongruent conditions, incongruent conditions and neutral conditions under all cue conditions, no significant differences were observed between congruent and neutral conditions under all cue conditions. There were no significant main effect of group $\left(F_{(1,63)}=0.03, p=0.87, \eta_{p}{ }^{2}=0.00\right)$, group and flanker type $\left(\mathrm{F}_{(2,126)}=1.59, \mathrm{p}=0.21, \eta_{\mathrm{p}}{ }^{2}=0.03\right)$, group $\times$ cue type $\left(\mathrm{F}_{(3,189)}=0.88, \mathrm{p}=0.45, \eta_{\mathrm{p}}{ }^{2}=0.01\right)$ or group $\times$ cue type $\times$ flanker type $\left(F_{(6,378)}=1.59, p=0.15, \eta_{p}{ }^{2}=0.02\right)$ interaction. The descriptive data of the mean accuracy and standard deviations of athlete and non-athlete group according to the cue and flanker type are shown in Table.3.

Table.3 Mean accuracy (\%) and standard deviations of athlete and non-athlete group according to cue and flanker type

\begin{tabular}{ccccccc}
\hline & \multicolumn{2}{c}{ Congruent } & \multicolumn{2}{c}{ Incongruent } & \multicolumn{2}{c}{ Neutral } \\
\cline { 2 - 6 } & Athlete & Non-athlete & Athlete & Non-athlete & Athlete & Non-athlete \\
\hline No cue & $96.4 \pm 4.5$ & $96.9 \pm 5.6$ & $94.8 \pm 4.3$ & $93.6 \pm 6.1$ & $99.0 \pm 2.8$ & $99.3 \pm 2.0$ \\
Central cue & $98.2 \pm 2.9$ & $97.8 \pm 3.7$ & $91.9 \pm 8.9$ & $94.5 \pm 6.7$ & $98.6 \pm 2.7$ & $99.1 \pm 2.2$ \\
Double cue & $99.0 \pm 2.3$ & $97.2 \pm 4.4$ & $94.4 \pm 6.7$ & $95.8 \pm 5.0$ & $98.8 \pm 2.5$ & $98.3 \pm 2.8$ \\
Spatial cue & $99.4 \pm 1.9$ & $98.0 \pm 3.7$ & $96.8 \pm 4.8$ & $97.6 \pm 5.3$ & $99.2 \pm 2.7$ & $99.3 \pm 2.0$ \\
\hline
\end{tabular}


208

209

210

211

212

\subsection{Differences of athletes and non-athletes on the 3 components of attentional network}

Independent samples t-tests were carried out for each component of the attentional system (alerting, orienting, and executive networks). Results showed a significant difference between athlete and non-athlete group on executive network $\left(\mathrm{t}_{(63)}=2.36, \mathrm{p}=0.02\right)$, while no differences were observed on alerting $\left(\mathrm{t}_{(63)}=\right.$ $0.05, \mathrm{p}=0.96)$ or orientation $\left(\mathrm{t}_{(63)}=-1.32, \mathrm{p}=0.19\right)$ networks (see Fig.2).

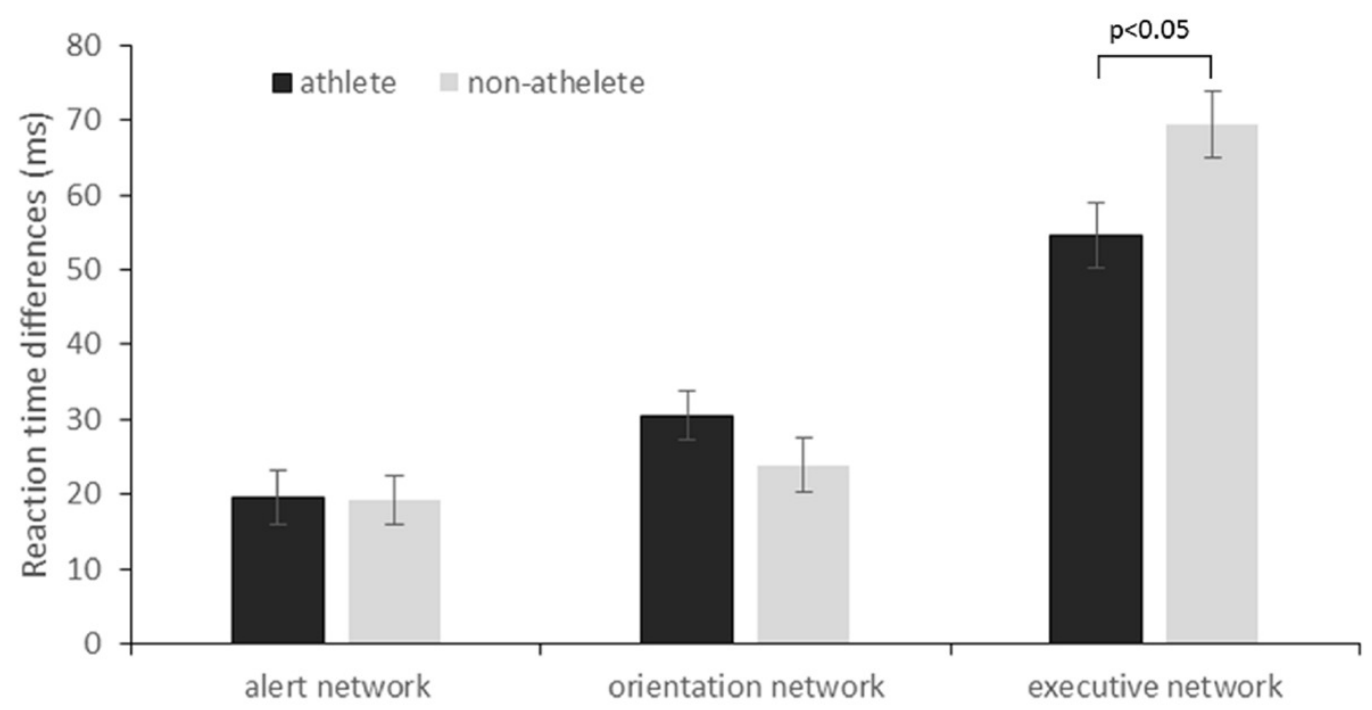

213

Fig.2 Reaction time differences that reflect the efficiency of the three attentional networks of athlete and non-athlete group (mean $\pm \mathbf{S E}$ ). The smaller differences on executive network and the larger differences on alerting and orientation network indicate a better function.

\section{Discussion}

The aim of the present study was to investigate the relationship between sports training experience and the attentional network using the ANT. Our results showed that the athlete group received a higher score than the non-athlete group on the executive network component, which is consistent with previous findings that have confirmed a positive correlation between executive control and athletic ability (Jacobson \& Matthaeus, 2014; Vestberg et al., 2012). A possible reason for the superior executive network function of athletes may be mainly due to the cognitive benefit of physical activity. Also, it has been proposed that exercises performed in the cognitively challenged environment are more effective to induce neural and cognitive benefits than exercise alone (Fabel et al., 2009). Table tennis athletes train and compete in the kind of enrichment environment that includes both physical and mental challenges. However, the present study cannot infer a causal relationship between athletic experience and attentional network function. It is possible that individuals who develop strong executive control skills are more likely to become athletes. Vestberg et al. (2012) suggest that individuals with 
229

230

231

232

233

234

235

236

237

238

239

240

241

242

243

244

245

246

247

248

249

250

251

252

253

254

255

256

257

258

259

260

261

262

263

264

265

266

high executive control ability become athletes more often and the ability further improved with training. It is speculated that the observed differences in attentional network may, at least in part, result from athletic participation.

The alerting and orientation of attention are especially important for athletes because they have to keep alerted all the time and orientate their attention quickly to the relevant information in the sporting context. However, the efficiency of the alerting and orientation networks tested by ANT did not differ in athletes and non-athletes in the present study. These results were inconsistent with previous findings, which have revealed that athletes practicing open-skilled sports showed superior ability on the alerting and voluntary orientation of attention than their counterbalanced controlled non-athlete group (Enns \& Richards, 1997; Nougier, Azemar, Stein, \& Ripoll, 1992). Both of these studies measured the alerting effect by testing more than one stimulus onset asynchrony (SOA) between cue and target, and the orienting effect was measured by comparing the reaction time difference between target stimuli at attended and unattended locations. However, the efficiency of alerting and orientation network tested by ANT were equivalent in athletes and non-athletes in the present study. This is consistent with the meta-analysis by Voss et al. (2010). They found the effects of athlete experience were small and not statistically significant $(\mathrm{g}=.17 ; \mathrm{p}>.05)$ in attentional cuing paradigm which is similar to the alerting and orienting network tests of the ANT in the present study. A possible reason for the inconsistency may be mainly due to the different experimental paradigms. The ANT used in this study is a relative simple task, and the response times for the measurement of orienting might have been affected by a ceiling effect. Also, the participants in the non-athlete group seemed to participate in regular physical exercise which could improve their cognitive function (Voss, Nagamatsu, Liu-Ambrose, \& Kramer, 2011).

The selective enhancement of the executive control network in athletes is similar to previous studies focused on the effect of chronic exercise or acute exercise on alerting, orientation, and executive control using a similar version of the ANT. Pérez et al. (2014) found a difference between active and passive participants on the executive network while no differences were observed on the alerting and orientation network. Along the same line, Chang et al. (2015) found that rather than eliciting general improvement, a single bout of acute exercise selectively enhanced executive control of attention.

The present study also revealed a significant interaction between flanker type and cue type, suggesting that the orientation cue was most effective when conflict resolution was required, while the alerting cue failed to increase the efficiency of executive control. It mirrored the pattern of interactions obtained in an earlier study with adults using the ANT (Fan et al., 2002). The interaction between group and flanker type was consistent with the result that athletes were more efficient on the executive network.

Some limitations existed in the present study. Firstly, the cross-sectional design revealed a possible relationship between athletic experience and the attentional network, but it can hardly conclude a causal relationship. Longitudinal studies are needed in the future. Further, this design did not allow for deep exploration of the cause of selective enhancement of executive control of attention. Also, all the athlete participants in the study were qualified as the National Player at Second Grade. Athletes in different sport levels (e.g. elite and novice) should be enrolled in a future study to specify the relationship between attentional network and expertise in sports. 
267

268

269

270

271

272

273

274

275

276

277

278

279

280

281

282

283

284

285

286

287

288

289

290

291

292

293

294

295

296

297

298

299

300

301

\section{Conclusion}

In conclusion, college table tennis athletes exhibited selective enhancement of execution control of attentional networks while no differences between athletes and non-athletes were observed in the alerting and orientation networks. It suggests the existence of certain association between sports training experiences and the modulation of the executive control network.

\section{Reference}

Allard, F., Brawley, L. R., Deakin, J., \& Elliot, F. (1989). The effect of exercise on visual attention performance. Human Performance, 2(2), 131-145.

Brainard, D. H. (1997). The Psychophysics Toolbox. Spatial Vision, 10(4), 433-436.

Cereatti, L., Casella, R., Manganelli, M., \& Pesce, C. (2009). Visual attention in adolescents: Facilitating effects of sport expertise and acute physical exercise. Psychology of Sport \& Exercise, 10(1), 136-145.

Chang, Y. K., Pesce, C., Chiang, Y. T., Kuo, C. Y., \& Fong, D. Y. (2015). Antecedent acute cycling exercise affects attention control: An ERP study using attention network test. Frontiers in Human Neuroscience, 9, 156.

Enns, J. T., \& Richards, J. C. (1997). Visual Attentional Orienting in Developing Hockey Players. Journal of Experimental Child Psychology, 64(2), 255-275.

Fabel, K., Wolf, S. A., Ehninger, D., Babu, H., Leal-Galicia, P., \& Kempermann, G. (2009). Additive effects of physical exercise and environmental enrichment on adult hippocampal neurogenesis in mice. Frontiers in Neuroscience, 3, 1-7.

Fan, J., McCandliss, B. D., Fossella, J., Flombaum, J. I., \& Posner, M. I. (2005). The activation of attentional networks. Neuroimage, 26(2), 471-479. doi: 10.1016/j.neuroimage.2005.02.004

Fan, J., McCandliss, B. D., Sommer, T., Raz, A., \& Posner, M. I. (2002). Testing the efficiency and independence of attentional networks. Journal of cognitive neuroscience, 14(3), 340-347. doi: 10.1162/089892902317361886

Heppe, H., Kohler, A., Fleddermann, M. T., \& Zentgraf, K. (2016). The Relationship between Expertise in Sports, Visuospatial, and Basic Cognitive Skills. Frontiers in Psychology, 7, 904. doi: 10.3389/fpsyg.2016.00904

Jacobson, J., \& Matthaeus, L. (2014). Athletics and executive functioning: How athletic participation and sport type correlate with cognitive performance. Psychology of Sport \& Exercise, 15(5), 521-527.

Liou, Y. M., Jwo, C. J., Yao, K. G., Chiang, L. C., \& Huang, L. H. (2008). Selection of appropriate Chinese terms to represent intensity and types of physical activity terms for use in the Taiwan version of IPAQ. Journal of Nursing Research, 16(4), 252-263.

Memmert, D. (2009). Pay attention! A review of visual attentional expertise in sport. Parasitology Today, 2(12), 360361.

Memmert, D., Simons, D. J., \& Grimme, T. (2009). The relationship between visual attention and expertise in sports. Psychology of Sport \& Exercise, 10(1), 146-151.

Nougier, V., Azemar, G., Stein, J. F., \& Ripoll, H. (1992). Covert orienting to central visual cues and sport practice relations in the development of visual attention. Journal of Experimental Child Psychology, 54(3), 315-333. 
302

303

304

305

306

307

308

309

310

311

312

313

314

315

316

317

318

319

320

321

322

323

324

325

326

327

328

329

330

331
Padulo, J., Pizzolato, F., Tosi, R. S., Migliaccio, G. M., Attene, G., Curcio, R., \& Moura, Z. A. (2015). Task complexity reveals expertise of table tennis players. Journal of Sports Medicine \& Physical Fitness, 56(1-2), 149-156.

Pérez, L., Padilla, C., Parmentier, F. B., \& Andrés, P. (2013). The Effects of Chronic Exercise on Attentional Networks. Plos One, 9(7), e101478.

Petersen, S. E., \& Posner, M. I. (2012). The attention system of the human brain: 20 years after. Annual Review of Neuroscience, 35, 73.

Posner, M. I., \& Petersen, S. E. (1990). The Attention System of the Human Brain. Annual Review of Neuroscience, 13(1), 25-42.

Posner, M. I., \& Fan, J. (2008). Attention as an organ system. Topics in integrative neuroscience, 31-61.

Raab, M., Masters, R. S. W., \& Maxwell, J. P. (2005). Improving the 'how' and 'what' decisions of elite table tennis players. Human Movement Science, 24(3), 326-344.

Tang, Y. Y., \& Posner, M. I. (2009). Attention training and attention state training. Trends in cognitive sciences, 13(5), 222-227. doi: 10.1016/j.tics.2009.01.009

Verburgh, L., Scherder, E. J., van Lange, P. A., \& Oosterlaan, J. (2014). Executive functioning in highly talented soccer players. Plos One, 9(3), e91254. doi: 10.1371/journal.pone.0091254

Vestberg, T., Gustafson, R., Maurex, L., Ingvar, M., \& Petrovic, P. (2012). Executive functions predict the success of top-soccer players. Plos one, 7(4), e34731.

Voss, M. W., Kramer, A. F., Basak, C., Prakash, R. S., \& Roberts, B. (2010). Are expert athletes 'expert' in the cognitive laboratory? A meta-analytic review of cognition and sport expertise. Applied Cognitive Psychology, 24(6), 812826.

Voss, M. W., Nagamatsu, L. S., Liu-Ambrose, T., \& Kramer, A. F. (2011). Exercise, brain, and cognition across the life span. Journal of Applied Physiology, 111(5), 1505-1513.

Wang, C. H., Chang, C. C., Liang, Y. M., Shih, C. M., Chiu, W. S., Tseng, P., ... \& Juan, C. H. (2013). Open vs. closed skill sports and the modulation of inhibitory control. PloS one, 8(2), e55773.

Williams, A. M., \& Davids, K. (1999). Visual perception and action in sport. E\&FN Spon. 
Figure 1

\section{Stimuli and experimental paradigm of Attention Network Test (ANT)}

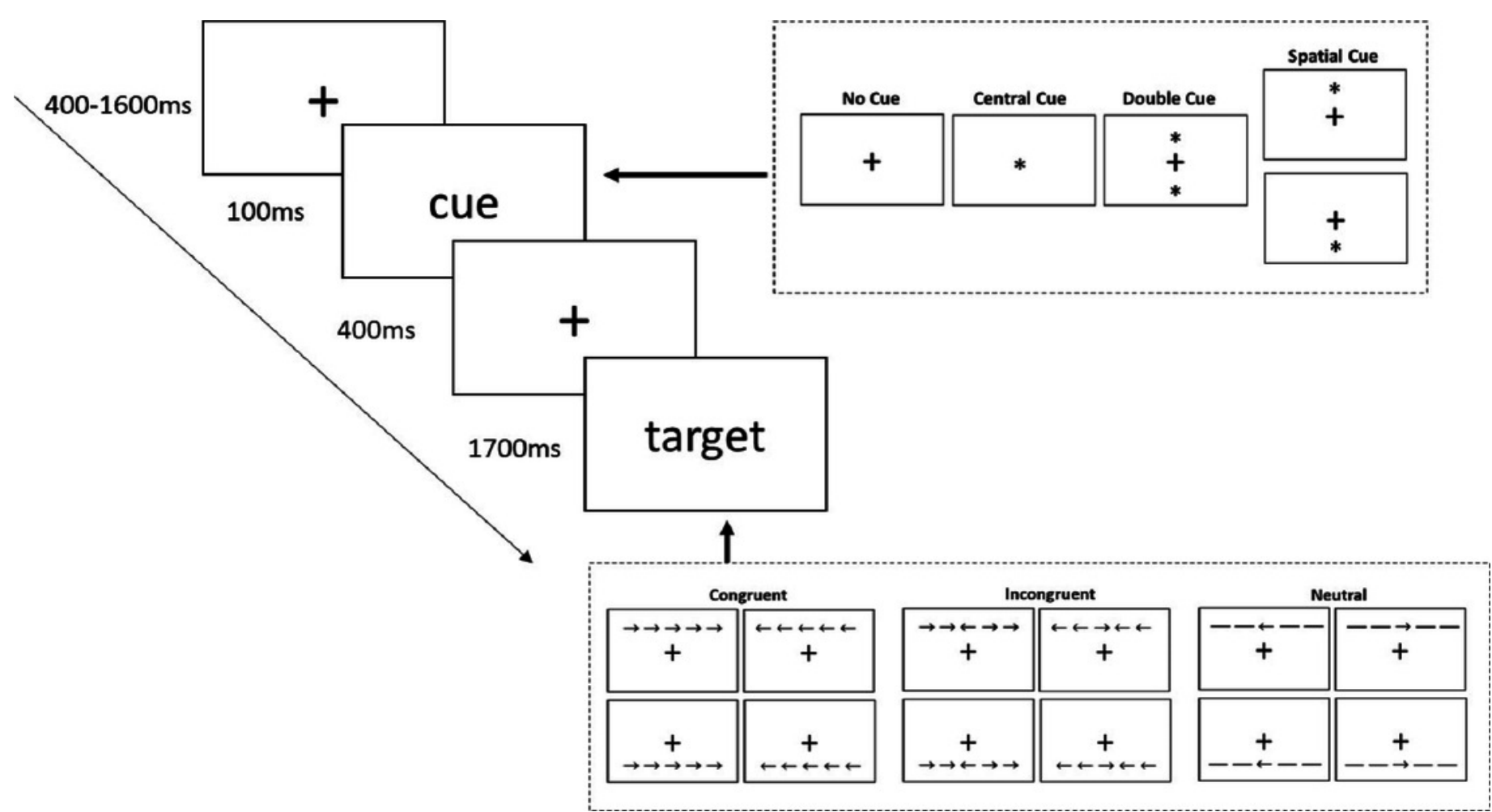




\section{Table 1 (on next page)}

The main characteristics of the subjects in different groups 
1

\begin{tabular}{|c|c|c|}
\hline & athlete group $(n=31)$ & non-athlete group $(n=34)$ \\
\hline Female & 11 & 14 \\
\hline Age (yr) & $21.90 \pm 1.72$ & $21.91 \pm 1.80$ \\
\hline Height (cm) & $1.73 \pm 0.08$ & $1.69 \pm 0.10$ \\
\hline Weight (kg) & $65.18 \pm 9.38$ & $61.13 \pm 9.67$ \\
\hline $\operatorname{BMI}\left(\mathrm{kg} / \mathrm{m}^{2}\right)$ & $21.69 \pm 1.72$ & $21.32 \pm 1.95$ \\
\hline \multicolumn{3}{|l|}{ IPAQ (METs/week) } \\
\hline Vigorous (METs/week) & $3587.09 \pm 2372.72$ & $2037.65 \pm 5109.58$ \\
\hline Moderate (METs/week) & $1597.42 \pm 1659.15$ & $927.06 \pm 1386.74$ \\
\hline Walking (METs/week) & $1448.47 \pm 1763.65$ & $1297.68 \pm 1261.23$ \\
\hline Overall (METs/week) & $6632.99 \pm 3808.16$ & $4262.38 \pm 5229.69 *$ \\
\hline Reaction time (ms) & $475.88 \pm 48.43$ & $488.45 \pm 34.94$ \\
\hline Accuracy (\%) & $97.93 \pm 1.93$ & $98.05 \pm 1.68$ \\
\hline
\end{tabular}

Table.1 The main characteristics of the subjects in different groups

2 Note. BMI=body mass index, IPAQ=International Physical Activity Questionnaire, METs=metabolic 3 equivalents. 


\section{Table 2 (on next page)}

Mean RTs (ms) and standard deviations of athlete and non-athlete group according to cue and flanker type 
1 Table.2 Mean RTs (ms) and standard deviations of athlete and non-athlete group according to cue and

2 flanker type

\begin{tabular}{ccccccc}
\hline & \multicolumn{2}{c}{ Congruent } & \multicolumn{2}{c}{ Incongruent } & \multicolumn{2}{c}{ Neutral } \\
\cline { 2 - 6 } & Athlete & Non-athlete & Athlete & Non-athlete & Athlete & Non-athlete \\
\hline No cue & $477.3 \pm 50.8$ & $486.1 \pm 36.2$ & $532.6 \pm 55.2$ & $554.0 \pm 49.4$ & $485.6 \pm 55.3$ & $492.0 \pm 42.9$ \\
Central cue & $455.8 \pm 49.0$ & $459.8 \pm 38.6$ & $520.2 \pm 63.5$ & $537.3 \pm 43.1$ & $458.4 \pm 49.4$ & $465.6 \pm 40.7$ \\
Double cue & $459.7 \pm 49.7$ & $465.0 \pm 41.1$ & $519.6 \pm 56.3$ & $542.0 \pm 43.5$ & $457.7 \pm 48.7$ & $467.4 \pm 43.2$ \\
Spatial cue & $434.0 \pm 52.0$ & $446.2 \pm 36.5$ & $472.3 \pm 51.6$ & $501.4 \pm 43.8$ & $435.9 \pm 48.1$ & $443.4 \pm 36.9$ \\
\hline
\end{tabular}

3

4 


\section{Table 3 (on next page)}

Mean accuracy (\%) and standard deviations of athlete and non-athlete group according to cue and flanker type 
1 Table.3 Mean accuracy (\%) and standard deviations of athlete and non-athlete group according to cue 2 and flanker type

\begin{tabular}{ccccccc}
\hline & \multicolumn{2}{c}{ Congruent } & \multicolumn{2}{c}{ Incongruent } & \multicolumn{2}{c}{ Neutral } \\
\cline { 2 - 6 } & Athlete & Non-athlete & Athlete & Non-athlete & Athlete & Non-athlete \\
\hline No cue & $96.4 \pm 4.5$ & $96.9 \pm 5.6$ & $94.8 \pm 4.3$ & $93.6 \pm 6.1$ & $99.0 \pm 2.8$ & $99.3 \pm 2.0$ \\
Central cue & $98.2 \pm 2.9$ & $97.8 \pm 3.7$ & $91.9 \pm 8.9$ & $94.5 \pm 6.7$ & $98.6 \pm 2.7$ & $99.1 \pm 2.2$ \\
Double cue & $99.0 \pm 2.3$ & $97.2 \pm 4.4$ & $94.4 \pm 6.7$ & $95.8 \pm 5.0$ & $98.8 \pm 2.5$ & $98.3 \pm 2.8$ \\
Spatial cue & $99.4 \pm 1.9$ & $98.0 \pm 3.7$ & $96.8 \pm 4.8$ & $97.6 \pm 5.3$ & $99.2 \pm 2.7$ & $99.3 \pm 2.0$ \\
\hline
\end{tabular}

3

4 
Figure 2

Reaction time differences that reflect the efficiency of the three attentional networks of athlete and non-athlete group (mean \pm SE)

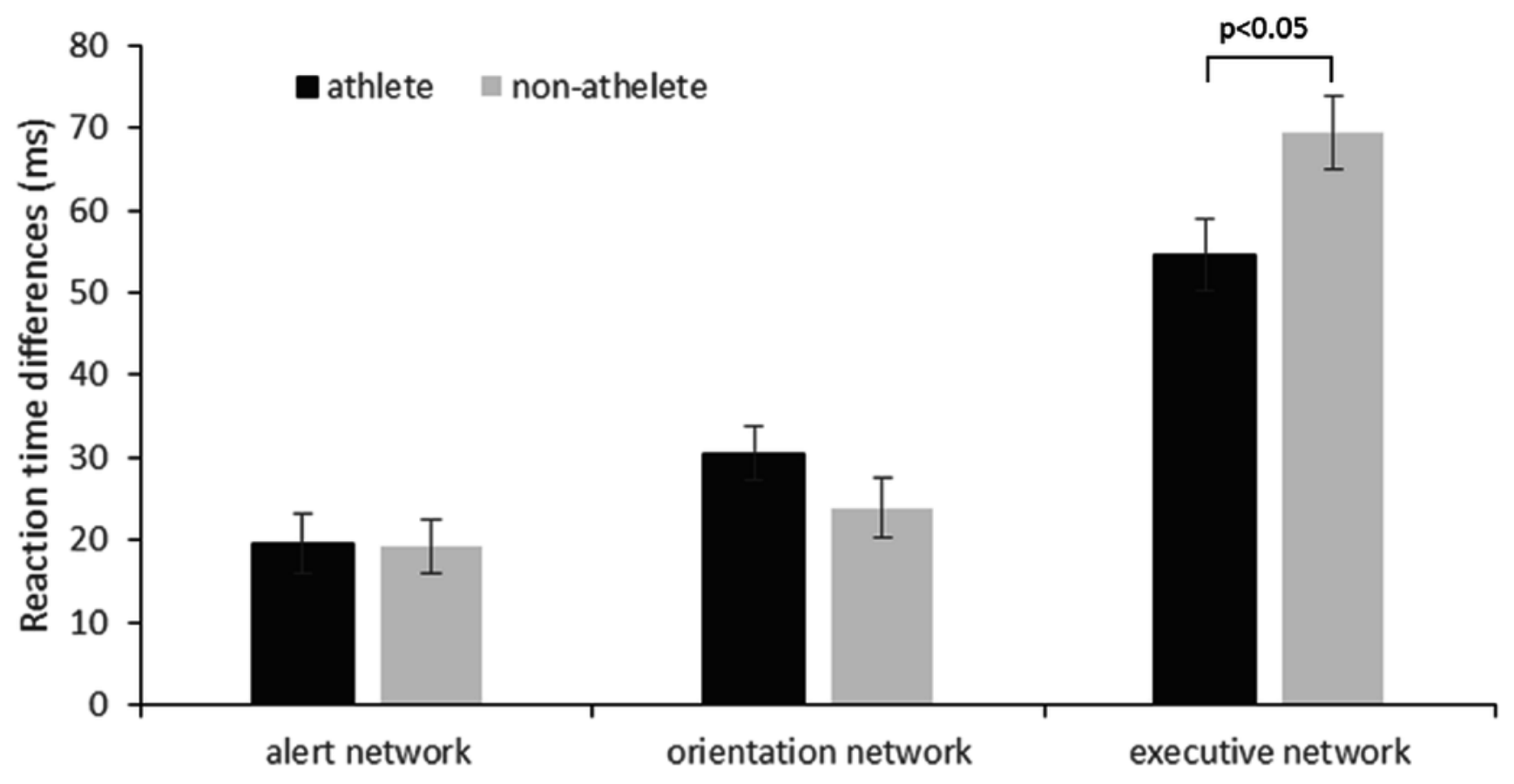

